\section{Adolescentes e suas relações com serviços de saúde: estudo transversal em escolares de Niterói, Rio de Janeiro, Brasil}

\author{
Adolescents and their relationship to health \\ services: a school-based cross-sectional study \\ in Niterói, Rio de Janeiro, Brazil
}

\author{
Lenita Barreto Lorena Claro 1 \\ Claudia March 1 \\ Monica Tereza Machado Mascarenhas ${ }^{1}$ \\ Isabel Antonia Barros de Castro ${ }^{2}$ \\ Maria Luiza Garcia Rosa 1
}

\author{
1 Instituto de Saúde da \\ Comunidade, Universidade \\ Federal Fluminense, \\ Niterói, Brasil. \\ 2 Universidade Federal \\ Fluminense, Niterói, Brasil. \\ Correspondência \\ M. L. G. Rosa \\ Instituto de Saúde da \\ Comunidade, Universidade \\ Federal Fluminense. \\ Rua Marquês de Paraná 303, \\ Prédio Anexo ao Hospital \\ Universitário Antônio Pedro, \\ 3o andar, Niterói, $R J$ \\ 24230-030, Brasil. \\ mluizagr@vm.uff.br
}

\begin{abstract}
This cross-sectional study describes the relationship between adolescents and health services using several indicators (self-reported diseases, self-rated health status, the need and search for health care, health care utilization and fidelity, adherence to medical prescription) and their association with socio-demographic variables. The adolescents (aged 12 to 17 years) were enrolled in schools from a district in Niteroi, Rio de Janeiro State, Brazil. Type of school, representing the participants' socioeconomic status, was associated with all the indicators. Adolescents in public schools tended to rate their health status as fair or poor. They also showed lower odds of reporting health needs, the search for health care, obtaining access, and remaining faithful to health services. Girls rated their health status as fair or poor and sought health care more frequently boys. High adherence to medical prescription of medicines and tests was observed in the sample.
\end{abstract}

Adolescent; Adolescent Health Services; Social Conditions

\section{Introdução}

Há alguns anos, a adolescência era o período de menor risco de adoecimento e morte. No entanto, nas duas últimas décadas, observouse um aumento da morbi-mortalidade nesse grupo populacional 1 . De um lado, pesquisas científicas têm revelado que várias alterações orgânicas, advindas de hábitos de vida pouco saudáveis, já estão instaladas nesse momento da vida, constituindo-se em fatores de risco para doenças, principalmente na vida adulta. De outro lado, comportamentos de risco são cada vez mais comuns, como conseqüência, principalmente, de tensões econômicas e sociais 2 . Um exemplo é a evolução do coeficiente de mortalidade por causas externas na população brasileira de 10 a 19 anos, que passou de 36,56 por $100 \mathrm{mil}$, em 1980, para 47,67, em 2002 (DATASUS. Indicadores de saúde: mortalidade e nascidos vivos, 2004. http://www.ibge.gov.br/ home/estatistica/populacao/trabalhoerendimento/pnad98/saude/apresentacao.shtm; acessado em 04/Dez/2004). Esse novo e inaceitável padrão de morbi-mortalidade se repete em várias partes do mundo e tem feito os sistemas de saúde se voltarem para pesquisar e criar programas que sejam capazes de atender às necessidades específicas dos adolescentes, o que tem se revelado um grande desafio 3,4 . Os adolescentes têm necessidades e expectativas peculiares, tendem a não valorizar sintomas 
que não sejam muito graves e que não sejam físicos 5 e tendem a não aderir tanto às ações de prevenção quanto às de tratamento $6,7,8$.

Apesar da relevância, a relação dos adolescentes com os serviços de saúde é pouco investigada no Brasil. O estudo dessa temática torna-se oportuno para orientar as ações de saúde voltadas para esse grupo populacional.

O presente estudo faz parte de uma pesquisa realizada nas escolas do bairro do Fonseca, no Município de Niterói, Rio de Janeiro, durante o período de outubro de 2003 até junho de 2004. Visa descrever a relação dos adolescentes com os serviços de saúde através dos indicadores - morbidade referida, auto-avaliação do estado de saúde, necessidade de saúde sentida, demanda, utilização, fidelidade aos serviços de saúde e adesão aos cuidados de saúde - e sua associação com variáveis sócio-demográficas.

\section{Material e métodos}

Trata-se de um estudo transversal, no qual foram avaliados os adolescentes entre 12 e 17 anos, de ambos os sexos, a partir de uma amostra proporcional ao número de alunos matriculados em escolas públicas e privadas do bairro estudado, por idade. A partir das listas do censo escolar de 2001 do INEP (Instituto Nacional de Estudos e Pesquisas Educacionais Anísio Teixeira), identificaram-se as escolas com cinqüenta e mais alunos matriculados na faixa etária estudada. Essas escolas foram visitadas, e as listagens de alunos por faixa etária e turma foram registradas e serviram de base para o sorteio da amostra de 480 alunos (400 mais $20 \%$ de perdas), calculada para o poder de $80 \%$ e significância estatística de 5\%, para identificar prevalência de $8 \%$ na população de estudo. Foram listados 4.530 alunos, e participaram do estudo 457 do total de matriculados, havendo 23 perdas, decorrentes de faltas que não puderam ser substituídas (20 casos) ou recusas (3 casos). Todos os participantes de 15 anos e mais assinaram o consentimento informado, e os participantes de 12 a 14 anos trouxeram o consentimento assinado pelo responsável. O projeto foi aprovado pelo Comitê de Ética do Hospital Universitário Antônio Pedro.

Aplicou-se um questionário com instrumentos validados e questões sócio-demográficas. As perguntas sobre a relação com os serviços de saúde basearam-se, em sua maioria, nos conceitos definidos para a pesquisa sobre acesso e utilização de serviços de saúde do IBGE (Instituto Brasileiro de Geografia e Estatística) que estão listados a seguir 2: (i) Morbidade referida - a referência a uma doença, crônica ou aguda, no momento da entrevista foi chamada de morbidade referida (na pesquisa do IBGE, foi considerada somente a presença de doença crônica); (ii) Auto-avaliação do estado de saúde - o estado de saúde do adolescente foi avaliado segundo seu próprio ponto de vista e foi classificado em uma escala de cinco graus: muito bom, bom, regular, ruim ou muito ruim; (iii) Necessidade sentida - consideramos necessidade sentida a referência à necessidade de buscar um serviço de saúde, independentemente da procura efetiva. Não há conceito análogo na pesquisa do IBGE; (iv) Demanda - chamamos de demanda a procura de atendimento de serviço de saúde, para qualquer motivo, independente de ter sido em um serviço formal ou informal; (v) Acesso - consideramos bom acesso quando houve atendimento logo na primeira vez da procura; (vi) Fidelidade - chamamos de fidelidade o hábito de procurar o mesmo profissional ou serviço quando precisava de atendimento de saúde, independente de ser um serviço formal ou informal; (vii) Utilização para o adolescente que, no período de referência, consultou médico ou foi internado, foi perguntado o número de vezes que procurou esse atendimento; (viii) Adesão - consideramos que houve adesão ao tratamento quando o adolescente, que recebeu uma prescrição do profissional de saúde, considerou tê-la seguido. Não há conceito análogo na pesquisa do IBGE. Optamos por manter o intervalo de três meses entre o evento relatado e a data do preenchimento do questionário para todas as questões sobre a relação dos adolescentes com os serviços de saúde, porque eles adoecem pouco. Tal período é inferior ao adotado no inquérito National Health Interview Survey Items in the Adult and Child, que é de 12 meses, e superior ao utilizado pela Pesquisa Nacional por Amostra de Domicílio (PNAD), que é de 15 dias 2,9. Quanto à idade, embora existam estudos mostrando que a confiabilidade é menor quando o respondente é menor de 14 anos, o estudo de Horiwtz et al. 10 suporta a decisão de incluir, na pesquisa, alunos de 12 e mais anos. Os autores, analisando a validade e a confiabilidade do instrumento Services Assessment for Children and Adolescents (SACA), para usuários de serviços de saúde mental, encontraram confiabilidade boa a excelente quando o instrumento foi administrado em crianças de 11 anos e mais, para uso durante toda a vida e nos últimos 12 meses. As demais variáveis do questionário selecionadas para análise foram sexo, faixa etária, tipo de escola (utilizada como proxy de condição sócio-econômica). 
O questionário foi autopreenchido na presença de pesquisadores treinados, os quais esclareceram as dúvidas. Foi realizado um estudo piloto em duas fases para testar a compreensão dos adolescentes sobre as questões incluídas.

\section{Análise estatística}

Foram estimadas as prevalências dos indicadores representados pelas respostas positivas e negativas às perguntas do questionário e realizada a análise bivariada de cada indicador com as variáveis selecionadas segundo a revisão da literatura. Realizou-se o teste do qui-quadrado de Pearson, com correção de Yates e significância de $5 \%$, para testar a hipótese de associação entre as variáveis. Calcularam-se os odds ratios (OR) não ajustados e seus intervalos de confiança. A seguir, foi realizada uma regressão logística para cada indicador com a estimação do OR ajustado, incluindo as variáveis que tiveram valores de $\mathrm{p}$ inferiores a 0,10 na análise bivariada correspondente, no intuito de controlar possíveis confundimentos.

\section{Resultados}

Foram entrevistados 457 adolescentes, sendo 254 (55,6\%) do sexo feminino e $203(44,4 \%)$ do sexo masculino. Do total, 328 adolescentes $(71,8 \%)$ estudavam em escolas públicas; e 129 $(28,2 \%)$, em escolas privadas; $220(48,1 \%)$ tinham entre 12 e 14 anos de idade; e $237(51,9 \%)$ tinham entre 15 e 17 anos.

Setenta e sete $(16,8 \%)$ dos entrevistados afirmaram fazer tratamento para alguma doença (morbidade referida) no momento da pesquisa. Destes, sessenta e dois apontaram o motivo do tratamento, havendo sete referências à bronquite/asma, duas à pneumonia, acne, alergia e anemia; os demais motivos foram citados somente uma vez, não havendo menção à hipertensão arterial.

Sobre a auto-avaliação do estado de saúde, 357 adolescentes $(78,1 \%)$ consideraram seu estado de saúde como muito bom ou bom; e 78 $(17,1 \%)$, como regular, ruim ou muito ruim; 22 adolescentes não responderam à questão $(4,8 \%)$. $\mathrm{Na}$ análise bivariada, as variáveis sexo, tipo de escola e morbidade referida apresentaram associação estatisticamente significativa com a auto-avaliação do estado de saúde e foram retidas para a regressão logística, que confirmou a significância e independência da associação. As meninas, os alunos de escolas públicas $\mathrm{e}$ aqueles que afirmaram estar tratando de uma doença no momento da pesquisa tiveram chan- ces maiores de ter uma auto-avaliação de saúde classificada como regular, ruim ou muito ruim (Tabela 1).

Quanto à necessidade sentida, 210 (46\%) adolescentes afirmaram ter sentido necessidade de procurar um serviço de saúde nos últimos três meses, e 228 (49,9\%) afirmaram não ter sentido tal necessidade; $19(4,2 \%)$ adolescentes não responderam à pergunta. $\mathrm{Na}$ análise bivariada, observou-se uma associação estatisticamente significativa com tipo de escola, morbidade referida, auto-avaliação do estado de saúde e sexo. Após a regressão logística, a associação com as três primeiras variáveis manteve a significância, sendo maior a chance de um aluno de escola privada, de um adolescente em tratamento de doença e daquele que avaliou seu estado de saúde como regular, ruim ou muito ruim afirmarem ter precisado de um serviço de saúde nos últimos três meses (Tabela 2).

No tocante à demanda a serviços de saúde, $218(47,7 \%)$ adolescentes disseram ter procurado um serviço de saúde nos últimos três meses; e 220 (48,1\%), não ter procurado; 19 (4,2\%) não responderam a essa questão. Na análise bivariada, as variáveis sexo, tipo de escola, morbidade referida, auto-avaliação do estado de saúde e necessidade sentida mostraram uma associação estatisticamente significativa com a demanda a serviços de saúde. Após o ajuste feito com a regressão logística, os alunos de escolas privadas, os adolescentes em tratamento de doença e aqueles que sentiram necessidade de procurar um serviço de saúde nos últimos três meses apresentaram maiores chances de procura de um serviço de saúde nesse período (Tabela 3).

Dos 210 adolescentes que afirmaram ter precisado de um serviço de saúde, $166(79,1 \%)$ responderam ter efetivamente procurado o serviço; 40 (19\%), não ter procurado; 4 (1,9\%) não responderam à pergunta. Dentre os que afirmaram ter precisado, mas não ter procurado um serviço de saúde, 30 apontaram o motivo: a doença não era considerada tão grave (12), preguiça (11), falta de tempo (3), o responsável não pôde levá-lo (3) e o serviço não funcionava (1).

Em referência ao acesso aos serviços de saúde, 190 (41,6\%) disseram não ter ido a nenhum serviço de saúde nos últimos três meses, $195(42,7 \%)$ afirmaram ter sido atendidos no primeiro serviço que buscaram, $51(11,2 \%)$ responderam que não foram atendidos no primeiro serviço que procuraram, da última vez que foram a um serviço de saúde, e 21 (4,6\%) não responderam à questão. Somente o tipo de escola mostrou associação estatisticamente significativa com o acesso. A chance de um aluno de escola pública não ter sido atendido no primei- 
Auto-avaliação do estado de saúde segundo o sexo, faixa etária, tipo de escola e morbidade referida.

\begin{tabular}{|c|c|c|c|c|c|c|c|c|}
\hline \multirow[t]{2}{*}{ Variável } & \multicolumn{2}{|c|}{$\begin{array}{l}\text { Regular, ruim } \\
\text { ou muito ruim }\end{array}$} & \multicolumn{2}{|c|}{$\begin{array}{l}\text { Muito bom } \\
\text { ou bom }\end{array}$} & \multirow[t]{2}{*}{$\begin{array}{l}\text { OR não ajustado } \\
\text { (IC95\%) }\end{array}$} & \multirow[t]{2}{*}{ p-valor } & \multirow[t]{2}{*}{$\begin{array}{l}\text { OR ajustado } \\
\text { (IC95\%) }\end{array}$} & \multirow[t]{2}{*}{$p$ valor } \\
\hline & $\mathrm{n}$ & $\%$ & $\mathrm{n}$ & $\%$ & & & & \\
\hline \multicolumn{9}{|l|}{ Sexo } \\
\hline Masculino & 24 & 12,4 & 170 & 87,6 & 1 & 0,01 & 1 & 0,004 \\
\hline Feminino & 54 & 22,4 & 187 & 77,6 & $2,05(1,18-3,57)$ & & $2,23(1,29-3,83)$ & \\
\hline \multicolumn{9}{|c|}{ Faixa etária (anos) } \\
\hline $12-14$ & 32 & 15,2 & 179 & 84,8 & 1 & 0,182 & - & \\
\hline $15-17$ & 46 & 20,5 & 178 & 79,5 & $1,45(0,86-2,45)$ & & - & - \\
\hline \multicolumn{9}{|c|}{ Tipo de escola } \\
\hline Privada & 12 & 10,1 & 107 & 89,9 & 1 & 0,013 & 1 & 0,005 \\
\hline Pública & 66 & 20,9 & 250 & 79,1 & $2,35(1,18-4,80)$ & & $2,64(1,35-5,17)$ & \\
\hline \multicolumn{9}{|c|}{ Morbidade referida } \\
\hline Não & 53 & 15,1 & 297 & 84,9 & 1 & & 1 & 0,001 \\
\hline Sim & 24 & 32,4 & 50 & 67,6 & $2,69(1,47-4,92)$ & $<0,001$ & $2,72(1,51-4,90)$ & \\
\hline
\end{tabular}

Tabela 2

Necessidade sentida segundo o sexo, faixa etária, tipo de escola, morbidade referida e auto-avaliação do estado de saúde e fidelidade.

\begin{tabular}{|c|c|c|c|c|c|c|c|c|}
\hline \multirow[t]{2}{*}{ Variável } & \multicolumn{2}{|c|}{ Sim } & \multicolumn{2}{|c|}{ Não } & \multirow{2}{*}{$\begin{array}{l}\text { OR não ajustado } \\
\text { (IC95\%) }\end{array}$} & \multirow[t]{2}{*}{ p-valor } & \multirow{2}{*}{$\begin{array}{l}\text { OR ajustado } \\
\text { (IC95\%) }\end{array}$} & \multirow[t]{2}{*}{$p$ valor } \\
\hline & $\mathrm{n}$ & $\%$ & $\mathrm{n}$ & $\%$ & & & & \\
\hline \multicolumn{9}{|l|}{ Sexo } \\
\hline Masculino & 82 & 42,5 & 11 & 57,5 & 1 & 0,053 & 1 & 0,36 \\
\hline Feminino & 128 & 52,2 & 117 & 47,8 & $1,48(0,99-2,21)$ & & $1,21(0,80-1,82)$ & \\
\hline \multicolumn{9}{|l|}{ Faixa etária (anos) } \\
\hline $12-14$ & 92 & 43,8 & 118 & 43,8 & 1 & 0,117 & - & \\
\hline $15-17$ & 118 & 51,8 & 110 & 51,8 & $1,38(0,93-2,04)$ & & - & - \\
\hline \multicolumn{9}{|l|}{ Tipo de escola } \\
\hline Privada & 74 & 59,7 & 50 & 40,3 & 1 & 0,003 & 1 & 0,001 \\
\hline Pública & 136 & 43,3 & 178 & 56,7 & $0,52(0,33-0,80)$ & & $0,47(0,30-0,74)$ & \\
\hline \multicolumn{9}{|l|}{ Morbidade referida } \\
\hline Não & 155 & 44,0 & 197 & 56,0 & 1 & & 1 & 0,007 \\
\hline Sim & 52 & 68,4 & 24 & 31,6 & $2,75(1,58-4,83)$ & $<0,001$ & $2,14(1,23-3,72)$ & \\
\hline \multicolumn{9}{|l|}{$\begin{array}{l}\text { Auto-avaliação do } \\
\text { estado de saúde }\end{array}$} \\
\hline Muito bom ou bom & 150 & 43,0 & 199 & 57,0 & 1 & $<0,001$ & $3,23(1,83-5,69)$ & $<0,001$ \\
\hline $\begin{array}{l}\text { Regular, ruim } \\
\text { ou muito ruim }\end{array}$ & 54 & 71,1 & 22 & 28,9 & $3,26(1,84-5,79)$ & & & \\
\hline
\end{tabular}


Demanda a serviços de saúde segundo o sexo, faixa etária, tipo de escola, morbidade referida e auto-avaliação do estado de saúde e necessidade sentida.

\begin{tabular}{|c|c|c|c|c|c|c|c|c|}
\hline \multirow[t]{2}{*}{ Variável } & \multicolumn{2}{|c|}{ Sim } & \multicolumn{2}{|c|}{ Não } & \multirow{2}{*}{$\begin{array}{l}\text { OR não ajustado } \\
\text { (IC95\%) }\end{array}$} & \multirow[t]{2}{*}{$p$-valor } & \multirow{2}{*}{$\begin{array}{l}\text { OR ajustado } \\
\text { (IC95\%) }\end{array}$} & \multirow[t]{2}{*}{$p$ valor } \\
\hline & $\mathrm{n}$ & $\%$ & $\mathrm{n}$ & $\%$ & & & & \\
\hline \multicolumn{9}{|l|}{ Sexo } \\
\hline Masculino & 82 & 42,9 & 109 & 57,1 & 1 & 0,001 & 1 & 0,100 \\
\hline Feminino & 136 & 55,1 & 111 & 44,9 & $1,63(1,09-2,43)$ & & $1,52(0,92-2,53)$ & \\
\hline \multicolumn{9}{|l|}{ Faixa etária (anos) } \\
\hline $12-14$ & 104 & 49,5 & 106 & 50,5 & 1 & 0,997 & - & \\
\hline $15-17$ & 114 & 50,0 & 114 & 50,0 & $1,02(0,69-1,51)$ & & - & - \\
\hline \multicolumn{9}{|l|}{ Tipo de escola } \\
\hline Privada & 54 & 74,0 & 19 & 26,0 & 1 & $<0,001$ & 1 & 0,004 \\
\hline Pública & 136 & 43,3 & 172 & 56,7 & $0,40(0,26-0,64)$ & & $0,44(0,25-0,77)$ & \\
\hline \multicolumn{9}{|l|}{ Morbidade referida } \\
\hline Não & 160 & 45,2 & 194 & 54,8 & 1 & $<0,001$ & 1 & 0,010 \\
\hline Sim & 54 & 74,0 & 19 & 26,0 & $3,45(1,90-6,30)$ & & $2,55(1,25-5,20)$ & \\
\hline \multicolumn{9}{|l|}{$\begin{array}{l}\text { Auto-avaliação do } \\
\text { estado de saúde }\end{array}$} \\
\hline Muito bom ou bom & 159 & 45,4 & 191 & 54,6 & 1 & 0,001 & 1 & 0,421 \\
\hline $\begin{array}{l}\text { Regular, ruim } \\
\text { ou muito ruim }\end{array}$ & 49 & 67,1 & 24 & 32,9 & $2,45(1,40-4,32)$ & & $1,32(0,67-2,63)$ & \\
\hline \multicolumn{9}{|l|}{ Necessidade sentida } \\
\hline Não & 47 & 21,0 & 177 & 79,0 & 1 & $<0,001$ & 1 & 0,000 \\
\hline $\operatorname{Sim}$ & 166 & 80,6 & 40 & 19,4 & $15,63(9,50-25,81)$ & & $14,14(8,53-23,45)$ & \\
\hline
\end{tabular}

ro serviço que procurou foi 4,39 vezes a chance de um aluno de escola privada (Tabela 4).

Dos 51 que afirmaram não ter sido atendidos, somente 16 apontaram o motivo, sendo citados o excesso de pacientes com longo período de espera, falta de médicos ou serviço fechado.

Quatorze adolescentes (3,1\%) afirmaram ter sido hospitalizados nos últimos três meses. Dos 116 adolescentes que disseram quantas vezes procuraram um serviço de saúde nesse período, 53 (46\%) o fizeram uma vez, e 63 (54\%), duas ou mais vezes. Os motivos de busca mais citados foram: dor de dente ou tratamento dentário por 19 adolescentes, gripe ou resfriado por 11 , cefaléia ou dor de cabeça por 7 , atestado de saúde por 5 , bronquite por 4 , sinusite por 4 , vacinação por 2 .

Os serviços de saúde procurados nos últimos três meses compuseram dois grupos; o primeiro incluiu as referências a uma unidade de saúde: hospital, posto de saúde, clínica ou farmácia; e o outro, as referências a profissionais específicos. Na regressão logística, somente o tipo de escola mostrou uma associação estatisticamente significativa e independente com o tipo de serviço utilizado, como variável dicotômica. A chance de um aluno de escola pública indicar uma unidade de saúde e não um profissional em particular foi 14 vezes a chance de um aluno de escola privada o fazer.

Duzentos e vinte quatro (49,3\%) adolescentes afirmaram nunca ter tido a sua pressão arterial verificada anteriormente. Nas escolas privadas, essa proporção atingiu $46,3 \%$ dos alunos de 12 a 14 anos e 16,1\% dos alunos de 15 a 17 anos; nas escolas públicas, atingiu $62,9 \%$ e $50,6 \%$, respectivamente.

Do total de adolescentes que responderam à pergunta sobre fidelidade, $267(64,2 \%)$ afirmaram ser fiéis, isto é, procurar sempre o mesmo serviço de saúde quando sentiam necessidade; 84 (20,2\%) afirmaram não ser fiéis; e 65 $(15,6 \%)$ afirmaram nunca ter sentido necessidade de procurar serviços de saúde. A análise bivariada revelou associação estatisticamente significativa da fidelidade apenas com o tipo de escola: os alunos das escolas privadas mos- 
Tabela 4

Acesso a serviços de saúde segundo o sexo, faixa etária, tipo de escola, morbidade referida e auto-avaliação do estado de saúde e necessidade sentida.

\begin{tabular}{|c|c|c|c|c|c|c|}
\hline \multirow[t]{3}{*}{ Variável } & \multicolumn{4}{|c|}{ Acesso } & \multirow{3}{*}{$\begin{array}{l}\text { OR não ajustado } \\
\text { (IC95\%) }\end{array}$} & \multirow[t]{3}{*}{$p$ valor } \\
\hline & \multicolumn{2}{|c|}{ Não } & \multicolumn{2}{|c|}{ Sim } & & \\
\hline & $\mathrm{n}$ & $\%$ & $\mathrm{n}$ & $\%$ & & \\
\hline \multicolumn{7}{|l|}{ Sexo } \\
\hline Masculino & 18 & 18,9 & 77 & 81,1 & 1 & 0,699 \\
\hline Feminino & 33 & 21,9 & 118 & 78,1 & $1,20(0,60-2,39)$ & \\
\hline \multicolumn{7}{|c|}{ Faixa etária (anos) } \\
\hline $12-14$ & 29 & 23,0 & 97 & 77,0 & 1 & 0,454 \\
\hline $15-17$ & 22 & 81,7 & 98 & 18,3 & $1,33(0,68-2,60)$ & \\
\hline \multicolumn{7}{|c|}{ Tipo de escola } \\
\hline Privada & 6 & 7,7 & 72 & 92,3 & 1 & 0,001 \\
\hline Pública & 45 & 26,8 & 123 & 73,2 & $4,39(1,69-12,07)$ & \\
\hline \multicolumn{7}{|c|}{ Morbidade referida } \\
\hline Não & 43 & 22,3 & 147 & 77,4 & 1 & 0,291 \\
\hline Sim & 8 & 14,8 & 46 & 85,2 & $1,68(0,70-4,19)$ & \\
\hline
\end{tabular}

traram-se quase duas vezes mais fiéis que os alunos das escolas públicas (Tabela 5).

Entre os adolescentes que tiveram uma consulta médica nos últimos três meses, 162 $(35,4 \%)$ afirmaram que o profissional solicitou a realização de algum exame. Entre esses, 106 $(65,4 \%)$ afirmaram ter realizado o(s) exame(s); e $22(17,2 \%)$, não ter realizado; 34 (21\%) não responderam à pergunta. Na análise bivariada, apenas o sexo mostrou associação estatisticamente significativa com a adesão à realização de exames, a qual, entretanto, não permaneceu após o ajuste.

Cento e vinte sete adolescentes, dentre os que afirmaram ter tido uma consulta médica nos últimos três meses, afirmaram que o médico prescreveu algum medicamento ou orientação (dieta, exercícios e outras mudanças no estilo de vida). Desses, 91 (71,6\%) consideraram ter seguido a prescrição; e 31 (24,4\%), não a ter seguido; 5 (4\%) não responderam à questão. As variáveis idade e tipo de orientação (medicamento versus dieta, exercícios e outras) mantiveram associação estatisticamente significativa com a adesão ao tratamento após ajuste. Os adolescentes de 12 a 14 anos e aqueles que receberam prescrição medicamentosa tiveram maiores chances de aderir às orientações.

\section{Discussão}

Este estudo utilizou uma amostra de alunos entre 12 e 17 anos, faixa em que, segundo o Censo Demográfico de 2000 para Niterói, $100 \%$ da população está matriculada na rede escolar. No bairro estudado, reside uma população de diferentes estratos sócio-econômicos, e a amostragem proporcional ao número de matriculados fortalece a validade externa do estudo.

No presente estudo, 16,8\% dos adolescentes declararam ter uma doença (crônica ou aguda) no momento da entrevista. Na PNAD, 13,3\% dos adolescentes de 14 a 19 anos declararam ter doença crônica, entendida como aquela que acompanhava a pessoa por um longo período de tempo, podendo ter fases agudas, momentos de piora ou melhora sensível. As diferenças na idade e principalmente na abordagem da doença podem justificar o maior percentual encontrado entre os adolescentes aqui estudados.

A auto-avaliação do estado de saúde tem sido usada como indicador importante sobre o comportamento da população com relação à busca por serviços de saúde devido à dificuldade de mensurar-se a necessidade de saúde 11 . Esta é considerada um conceito impreciso, representado na literatura por uma gama de medidas que podem ser agrupadas em duas grandes categorias: o estado de saúde percebido individualmente e o avaliado pelo profissional. A auto-avaliação de saúde mostrou ser um preditor independente e consistente de morbidade e mortalidade 12, em diferentes grupos étnicos 13, estando associada com gênero e condição sócio-econômica 14 - mulheres e estratos desfavorecidos da população têm, em geral, pior auto-avaliação, inclusive em adolescentes 15 .

O percentual de adolescentes que avaliaram seu estado de saúde como regular, ruim ou muito ruim no presente estudo foi superior ao encontrado pela PNAD e em estudo com adolescentes americanos, ambos na faixa de 14 a 19 anos $(17,1 \%$ versus $8,76 \%$ e $8 \%$, respectivamente) 2,16. O sexo feminino, como em outras investigações, mostrou-se associado a uma pior avaliação do estado de saúde 2 . A chance de um aluno da escola pública avaliar seu estado de saúde como regular ou ruim foi maior que a de um aluno da escola privada, provavelmente em função do fator sócio-econômico, que representa um dos principais fatores de risco para o adoecimento, não só entre adolescentes, como nas demais faixas etárias 17. A iniqüidade também foi identificada como uma explicação para pior auto-avaliação do estado de saúde. Blakely et al. 18 observaram que pessoas vivendo em áreas de alta, média-alta e média-baixa ini- 
qüidade de renda tiveram OR regular/ruim de 1,20 (IC95\%: 1,041-1,38), 1,07 (0,95-1,25) e 1,02 (0,91-1,15), respectivamente, em comparação com pessoas residentes em áreas metropolitanas de menor desigualdade de renda.

Chama a atenção a baixa associação encontrada entre morbidade referida, auto-avaliação do estado de saúde e necessidade sentida. Enquanto apenas $16 \%$ dos entrevistados referiram estar se tratando de alguma doença no momento da pesquisa e mais de $82 \%$ avaliaram seu estado de saúde como muito bom ou bom, cerca de $48 \%$ afirmaram ter sentido necessidade de procurar um serviço de saúde nos últimos três meses. Essas discrepâncias podem traduzir uma limitação do questionário, uma vez que a morbidade referida e a auto-avaliação do estado de saúde se referiam ao momento da pesquisa e a necessidade sentida aos últimos três meses. Podem também indicar que a necessidade de buscar serviços de saúde, especialmente nessa faixa etária, não está vinculada, preferencialmente, à questão da morbidade, mas a ações preventivas, ou apontar para especificidades na abordagem do adolescente em relação ao conceito de saúde/doença.

De fato, a auto-avaliação do estado de saúde tem se mostrado associada à avaliação clínica quanto à presença ou ausência de uma condição crônica, sendo encontradas taxas de concordância de até $80 \% 19$. Por outro lado, estudos indicam que é também influenciada pela oferta de serviços e por fatores capacitantes, como, por exemplo, a situação sócio-econômica 20. No presente estudo, observa-se, claramente, a influência do fator capacitante quanto à necessidade sentida. A chance ajustada de um aluno de escola pública ter uma auto-avaliação de saúde regular, ruim ou muito ruim foi 2,64 vezes a de um aluno de escola privada. Em relação à expressão da necessidade, aconteceu o inverso: a chance ajustada de um aluno da escola pública foi 0,47 a chance de um aluno da escola privada, ou seja, para cada aluno da escola pública que afirmou ter sentido necessidade de procurar um serviço de saúde, houve dois alunos da escola privada que fizeram tal afirmação, independentemente do sexo, da idade, da referência à presença de doença ou da auto-avaliação do estado de saúde.

O conceito de utilização ou uso compreende o contato com os serviços de saúde. A utilização é resultante da interação do comportamento do indivíduo que demanda cuidados e do profissional que orienta o "doente" através do sistema de saúde (Andersen, 1968, apud Hulka $\&$ Wheat 21). Alguns estudos ressaltam a diferença de utilização segundo o gênero, sendo
Tabela 5

Fidelidade a serviços de saúde segundo o sexo, faixa etária, tipo de escola, morbidade referida e auto-avaliação do estado de saúde e necessidade sentida.

\begin{tabular}{|c|c|c|c|c|c|c|}
\hline \multirow[t]{3}{*}{ Variável } & \multicolumn{4}{|c|}{ Fidelidade } & \multirow{3}{*}{$\begin{array}{l}\text { OR não ajustado } \\
\text { (IC95\%) }\end{array}$} & \multirow[t]{3}{*}{$p$ valor } \\
\hline & \multicolumn{2}{|c|}{ Não } & \multicolumn{2}{|c|}{ Sim } & & \\
\hline & $\mathrm{n}$ & $\%$ & $\mathrm{n}$ & $\%$ & & \\
\hline \multicolumn{7}{|l|}{ Sexo } \\
\hline Masculino & 37 & 24,3 & 115 & 75,7 & 1 & 0,975 \\
\hline Feminino & 47 & 23,6 & 152 & 76,4 & $0,96(0,57-1,62)$ & \\
\hline \multicolumn{7}{|c|}{ Faixa etária (anos) } \\
\hline $12-14$ & 42 & 24,1 & 132 & 75,9 & 1 & 0,972 \\
\hline $15-17$ & 42 & 23,7 & 135 & 76,3 & $0,98(0,58-1,64)$ & \\
\hline \multicolumn{7}{|c|}{ Tipo de escola } \\
\hline Privada & 17 & 15,2 & 95 & 84,8 & 1 & 0,010 \\
\hline Pública & 67 & 28,0 & 172 & 72,0 & $2,18(1,17-4,10)$ & \\
\hline \multicolumn{7}{|c|}{ Morbidade referida } \\
\hline Não & 64 & 22,6 & 219 & 77,4 & 1 & 0,400 \\
\hline Sim & 18 & 28,6 & 45 & 71,4 & $1,37(0,71-2,63)$ & \\
\hline
\end{tabular}

maior nas mulheres em função da maior freqüência de uma auto-avaliação do estado de saúde menos positiva 22,23 , da maior preocupação das mulheres em relação à sua saúde, além dos eventos relacionados à maternidade 24 .

Alguns estudos encontraram uma maior morbidade feminina vinculada ao estresse psicológico 25,26 e uma maior tendência das mulheres, em comparação aos homens, de demandar atenção à saúde em função de fatores psicológicos 27. Settertobulte \& Kolip 23, em estudo com adolescentes alemães, encontrou que os meninos são menos propensos a revelarem seus problemas para os outros e a pedirem ajuda, ao contrário das meninas. No entanto, os autores não encontraram diferenças nas taxas de utilização de consultas gerais.

A análise da demanda a serviços de saúde mostrou forte associação com a necessidade sentida (OR ajustado de 14,14). As mulheres apresentaram maior demanda, embora a associação do gênero, depois do ajuste para faixa etária, tipo de escola, morbidade referida, auto-avaliação do estado de saúde e necessidade sentida, não tenha permanecido estatisticamente significativa. A chance de um aluno de escola pública demandar um serviço foi menos da metade do que a chance de um aluno de escola privada (OR ajustado de 0,44 ), independentemente do sexo, morbidade referida, auto-avaliação do estado de saúde e necessidade senti$\mathrm{da}$, mostrando que os grupos populacionais 
com melhor condição sócio-econômica demandam mais tais serviços, provavelmente em função da facilidade de acesso e outras questões culturais e sociais, como apontado por outros estudos 28,29. Apenas um, dos trinta adolescentes que apontaram o motivo da não procura do serviço do qual afirmaram ter precisado, alegou dificuldade de acesso.

O acesso aos serviços de saúde é um conceito que incorpora várias dimensões, sendo ele mesmo uma dimensão da qualidade. Visa mensurar a adequação, oportunidade dos serviços ofertados e as barreiras que possam dificultar a utilização do serviço demandado direta ou indiretamente pelo usuário 24 .

Em nossa pesquisa, o percentual de alunos que disseram não ter sido atendidos no primeiro serviço que procuraram foi de $11,2 \%$ e, na pesquisa no PNAD, na faixa de 5 a 19 anos, foi de $2,14 \% 2$. Os motivos citados, nas duas pesquisas, são muito semelhantes: não conseguiram vaga ou senha, não havia médicos em atividades de atendimento, não havia disponibilidade de serviço ou profissional especializado, o serviço ou equipamento não estava funcionando ou o tempo de espera foi muito longo, forçando à desistência. $\mathrm{O}$ tipo de escola foi a única variável, dentre as analisadas, que mostrou associação estatisticamente significativa com o acesso, sendo a chance de um aluno de escola pública não ter sido atendido no primeiro serviço que procurou, o dobro da chance de um aluno de escola privada, situação similar à descrita em estudo americano 29.

A relação do acesso com a demanda/utilização de serviços por adolescentes e a influência de outros fatores têm sido descritas na literatura. Um estudo com adolescentes de diversas classes sociais, em uma cidade americana com programas de inclusão social em serviços de saúde, mostrou que, apesar das facilidades para o acesso, os mais desfavorecidos buscavam e utilizavam menos os serviços de saúde, em função de barreiras não financeiras 30 . Um outro estudo a respeito de um programa de vacinação contra hepatite $\mathrm{B}$, desenvolvido em escolas do Estado americano do Texas no período 1998-2001, mostrou que, mesmo quando as barreiras econômicas são eliminadas, o gênero e a raça, traduzindo diferentes idéias culturais sobre saúde, podem interferir na demanda por serviços. Nesse estudo, houve maior participação no programa das meninas, assim como dos hispânicos e dos negros 31 .

O percentual de internações, no presente estudo, foi de $3,1 \%$, semelhante ao encontrado pela pesquisa do PNAD, na faixa de 5 a 19 anos, que foi de $3,6 \% 2$. Em relação ao motivo da pro- cura por um serviço, como na pesquisa do PNAD, a maior freqüência foi relacionada a tratamento odontológico, e os atestados de saúde foram citados por cerca de $2 \%$ dos entrevistados nas duas pesquisas.

O percentual de adolescentes que nunca tiveram sua pressão medida foi maior entre os alunos das escolas públicas, indicando uma baixa cobertura desse tipo de rastreamento. Com o conhecimento acumulado sobre o risco cardiovascular da hipertensão na adolescência, sabe-se que tal lacuna pode ter um grande impacto na saúde futura dessa população.

Abordando a fidelidade ao serviço de saúde, observou-se que a chance de um aluno da escola pública procurar de cada vez um serviço diferente, ou seja, não ser fiel, foi duas vezes a chance de um aluno da escola privada, o que destaca a relação da fidelidade com a melhor condição sócio-econômica desses adolescentes e sua conseqüente facilidade de acesso a serviços, muito provavelmente de natureza privada, por meio de planos e seguros de saúde. Scitovsky et al. 32 observaram que a falta de identificação, pelo paciente, de um médico específico em alguns planos de saúde retardava o cuidado e resultava em baixa freqüência de retorno às consultas. No presente estudo, o aluno de escola pública apresentou uma chance de procurar uma unidade de saúde, sem individualizar um profissional ou mesmo um serviço, quase cinco vezes a chance de um aluno de escola privada.

A adesão à realização de exames solicitados por médicos mostrou-se elevada $(82,8 \%)$ na amostra investigada. A adesão ao tratamento prescrito foi menor que a adesão à realização de exames, porém também se mostrou elevada (74,6\%), particularmente em relação aos tratamentos medicamentosos. Esses resultados colocam em questionamento a imagem freqüente do adolescente como pouco aderente às recomendações médicas 7,8 .

Os resultados do presente estudo, ao permitirem uma maior compreensão do papel de algumas variáveis no comportamento relacionado à utilização de serviços de saúde por adolescentes, representam uma contribuição às políticas e programas desenhados para aprimorar a qualidade da atenção à saúde a esse grupo populacional. 


\section{Resumo}

Este estudo transversal, realizado nas escolas de um bairro do Município de Niterói, Rio de Janeiro, Brasil, descreve a relação de adolescentes, entre 12 e 17 anos, com os serviços de saúde, através dos indicadores morbidade referida, auto-avaliação do estado de saúde, necessidade de saúde sentida, demanda, utilização, fidelidade aos serviços de saúde e adesão aos cuidados de saúde - e sua associação com variáveis sóciodemográficas. O nível sócio-econômico, representado pelo tipo de escola, mostrou-se associado a todos os indicadores. Os alunos das escolas públicas tinham uma chance maior do que os alunos das escolas privadas de avaliarem seu estado de saúde como regular ou ruime uma chance menor de expressarem necessidade sentida positivamente, demandarem serviços de saúde, obterem acesso aos serviços procurados e manterem-se fiéis aos serviços utilizados. As meninas apresentaram maior chance de auto-avaliarem seu estado de saúde como ruim ou muito ruim e de demandarem serviços de saúde. A adesão, tanto à realização de exames quanto ao tratamento prescrito por médicos, mostrouse elevada na amostra investigada.

Adolescente; Serviços de Saúde para Adolescentes; Condições Sociais

\section{Referências}

1. Shenkman E, Youngblade L, Nackashi J. Adolescents' preventive care experiences before entry into the State Children's Health Insurance Program (SCHIP). Pediatrics 2003; 112 (6 Pt 2):e533.

2. Instituto Brasileiro de Geografia e Estatística. Pesquisa nacional por amostra de domicílios. Acesso e utilização de serviços de saúde. 1998. http:// www.ibge.gov.br/home/estatistica/populacao/tr abalhoerendimento/pnad98/saude/apresentacao.shtm (acessado em 04/Dez/20004).

3. Kang M, Bernard D, Booth M, Quine S, Alperstein G, Usherwood T, et al. Access to primary health care for Australian young people: service provider perspectives. Br J Gen Pract 2003; 53:947-52.

4. Juszczak L, Cooper K. Improving the health and well-being of adolescent boys. Crit Care Nurs Clin North Am 2002; 37:433-42.

5. Palazzo LS, Béria JU, Tomasi E. Adolescentes que utilizan servicios de atención primaria: ¿Cómo viven? ¿Por qué buscan ayuda y cómo se expresan? Cad Saúde Pública 2003; 19:1655-65.

6. Booth ML, Bernard D, Quine S, Kang MS, Usherwood T, Alperstein G, et al. Access to health care among Australian adolescents young people's perspectives and their sociodemographic distribution. J Adolesc Health 2004; 34:97-103.

7. National Center for Chronic Disease Prevention and Health Promotion. Healthy youth. Health topics. http://www.cdc.gov/healthyyouth/health topics/index.htm (acessado em 04/Dez/2004).

\section{Colaboradores}

L. B. L. Claro participou da revisão bibliográfica, do desenho do estudo, da análise e discussão dos dados. C. March e M. L. G. Rosa contribuíram na revisão bibliográfica, no desenho do estudo, na coleta, análise e discussão dos dados. M. T. M. Mascarenhas participou da análise e da discussão dos dados. I. A. B. Castro participou da coleta e da análise dos dados.
8. Staples B, Bravender T. Drug compliance in adolescents: assessing and managing modifiable risk factors. Paediatr Drugs 2002; 4:503-13.

9. Centers for Disease Control and Prevention. National health interview survey. http://www.cdc. gov/nchs/about/major/nhis/hisdesgn.htm (acessado em 04/Dez/2004).

10. Horwitz SM, Hoagwood K, Stiffman AR, Summerfeld T, Weisz JR, Costello EJ, et al. Reliability of the services assessment for children and adolescents. Psychiatr Serv 2001; 52:1088-94.

11. Hulka BS, Wheat JR. Patterns of utilization. The patient perspective. Med Care 1985; 23:438-60.

12. Idler EL, Benyamini Y. Self-rated health and mortality: a review of twenty-seven community studies. J Health Soc Behav 1997; 38:21-37.

13. Chandola T, Jenkinson C. Validating self-rated health in different ethnic groups. Ethn Health 2000; 5:151-9.

14. Bobak M, Pikhart H, Rose R, Hertzman C, Marmot M. Socioeconomic factors, material inequalities, and perceived control in self-rated health: cross-sectional data from seven post-communist countries. Soc Sci Med 2000; 51:1343-50.

15. Goodman E. The role of socioeconomic status gradients in explaining differences in US adolescents' health. Am J Public Health 1999; 89: 1522-8.

16. Klein JD, McNulty M, Flatau CN. Adolescents' access to care: teenagers' self-reported use of ser- 
vices and perceived access to confidential care. Arch Pediatr Adolesc Med 1998; 152:676-82.

17. Dachs J, Norberto W. Determinantes das desigualdades na auto-avaliação do estado de saúde no Brasil: análise dos dados da PNAD/1998. Ciênc Saúde Coletiva 2002; 7:641-57.

18. Blakely TA, Lochner K, Kawachi I. Metropolitan area income inequality and self-rated health - a multi-level study. Soc Sci Med 2002; 54:65-77.

19. Viacava F. Informações em saúde: a importância dos inquéritos populacionais. Ciênc Saúde Coletiva 2002; 7:607-21.

20. Lambrew JM, DeFriese, GH, Carey TS, Ricketts TC, Biddle AK. The effects of having a regular doctor on access to primary care. Med Care 1996; 34:138-51.

21. Hulka BS, Wheat JR. Patterns of utilization. Med Care 1985; 23:438-60.

22. Settertobulte W, Kolip P. Gender-specific factors in the utilization of medical services during adolescence. J Adolesc 1997; 20:121-32.

23. Pinheiro RS, Viacava F, Travassos C, Brito AS Gênero, morbidade, acesso e utilização de serviços de saúde no Brasil. Ciênc Saúde Coletiva 2002; 7:687-707.

24. Pinheiro RS, Escosteguy CC. Epidemiologia e serviços de saúde. In: Medronho RA, organizador. Epidemiologia. Rio de Janeiro: Editora Atheneu; 2002. p. 361-9.
25. Bird CE, Rieker PP. Gender matters: an integrated model for understanding men's and women's health. Soc Sci Med 1999; 48:745-55.

26. Macintyre S, Ford G, Hunt K. Do women 'over-report' morbidity? Men's and women's responses to structured prompting on a standard question on long standing illness. Soc Sci Med 1999; 48:89-98.

27. Briscoe ME. Sex differences in perception of illness and expressed life satisfaction. Psychol Med 1978; 8:339-45.

28. Travassos C, Viacava F, Pinheiro R, Brito A. Utilization of health care services in Brazil: gender, family characteristics, and social status. Rev Panam Salud Publica 2002; 11:365-73.

29. Newacheck PW, Hughes DC, Stoddard JJ. Children's access to primary care: differences by race, income, and insurance status. Pediatrics 1996; 97:26-32.

30. Newacheck PW, Hung YY, Park MJ, Brindis CD, Irwin Jr. CE. Disparities in adolescent health and health care: does socioeconomic status matter? Health Serv Res 2003; 38:1235-52.

31. Middleman AB. Race/ethnicity and gender disparities in the utilization of a school-based hepatitis B immunization initiative. J Adolesc Health 2004; 34:414-9.

32. Scitovsky AA, Benham L, McCall N. Use of physician services under two prepaid plans. Med Care 1979; 17:441.

Recebido em 28/Jan/2005

Versão final reapresentada em 25/Nov/2005 Aprovado em 06/Dez/2005 